\title{
Prostatic calcifications are associated with a more severe symptom burden in men with type II chronic bacterial prostatitis
}

\author{
Konstantinos Stamatiou ${ }^{1}$, Vittorio Magri ${ }^{2}$, Gianpaolo Perletti ${ }^{3}$, Alberto Trinchieri ${ }^{4}$, Richard Lacroix ${ }^{1}$, \\ Nektaria Rekleiti ${ }^{1}$, Hippocrates Moschouris ${ }^{1}$ \\ ${ }^{1}$ Tzaneio Hospital, Piraeus, Greece; \\ ${ }^{2}$ ASST Nord Milano, Italy; \\ ${ }^{3}$ Department of Biotechnology and Life Sciences, Section of Medical and Surgical Sciences, University of Insubria, Varese, Italy; \\ ${ }^{4}$ CDC Ambrosiana Cesano B, Milano, Italy.
}

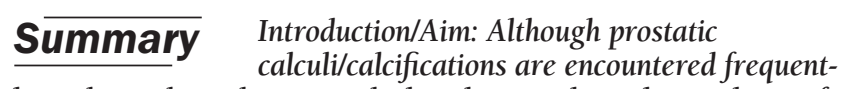
ly in the urological practice, little is known about the incidence of such lesions, their mechanism of formation, their relationship to other prostate conditions and their clinical significance. The purpose of this study is to describe the characteristics and to investigate the clinical significance of prostatic calcifications (PCs) in patients with chronic bacterial prostatitis (CBP).

Materials and methods: This study was conducted between 01/02/2013 and 20/02/2018. The patient population for this study included subjects with or without PCs and a confirmed diagnosis of NIH category II Chronic Bacterial Prostatitis (CBP). Demographics and clinical history of each assessed patient were reviewed. Eligible patients underwent prostatic ultrasound with post-void residual measurement, and the Meares-Stamey "4-glass" test. Symptom severity was measured using the National Institutes of Health Chronic Prostatitis Symptom Index (NIH-CPSI) and the International Prostatic Symptoms Score (IPSS). Antimicrobials were administered to confirmed cases of CBP according to the results of susceptibility tests. After four weeks off-therapy, the NIH-CPSI and IPSS tests were repeated. Variables were compared between patients with and without prostatic calcifications. Results: Ninety-five CBP patients were included in the study. According to the presence of PCs detected by ultrasound examination, patients were divided into two groups: 41 had PCs (group 1) and 54 didn't (group 2). No significant between-group baseline differences were found regarding age, marital status, prostate volume, the proportion of common CBP pathogens. Concerning highrisk sexual behavior, a significantly higher number of men with PCs practiced anal penetration. Moreover, a significantly higher number of men with PCs had a history of chronic prostatitis relapsing episodes. Microbiological eradication and the complete resolution of clinical symptoms occurred in similar proportions between the two groups. However, intergroup analysis resulted in significantly higher scores of the NIH-CPSI test in group 1, both at the pre-therapy and at the post-therapy time points. Conversely, no IPSS score differences between groups 1 and 2 were found at both pre- and post-therapy time points.

Conclusions: Prostatic calcifications do not seem to influence the microbiological outcome of antibacterial treatment. However, the CBP symptoms appear to be more severe in carriers of prostatic calcifications, either before or after antibacterial therapy.

KEY WORDS: Prostate; Prostatitis; Chronic bacterial prostatitis; Calcifications; Calculi; Stones.

Submitted 20 February 2019; Accepted 11 March 2019

\section{INTRODUCTION}

The terms prostatic calcifications (PCs), prostatic stones and prostatic calculi are used to describe hyperechoic calcium deposits within the prostate gland. They are a relatively common ultrasound finding whose pathophysiology is partially understood. However, the clinical relevance of these lesions and their association with prostatic diseases remains unclear. Traditionally, calcifications are considered to be a random finding of no clinical significance, probably associated with previous infection of the prostate. In fact, are usually found incidentally and are often not associated with a history of prostatitis. While histopathologic investigation showed that most calculi are associated with inflammatory changes, many of the relevant studies haven't correlated the presence of prostate calcifications with chronic bacterial prostatitis (CBP) (1). Nowadays the relation between PCs and CBP remains uncertain and it is still unknown if PCs are clinically insignificant or whether they have the potential to affect the treatment outcome. However, it is deemed important for specialists to become familiar with this entity. In this prospective, observational study, we wished to characterize the clinical features of PCs in men with chronic bacterial prostatitis (NIH Category II CBP) and to assess the outcome of therapy in order to better understand their impact on CBP. Comparison between CBP patients with or without signs of prostatic calcifications was also attempted.

\section{Patients AND Methods}

This study was conducted between 01/02/2013 and 20/02/2018, after approval by the local Ethics Committee. Participants enrolled for this study were first-referral urological male outpatients presenting with CBP symptoms. Group 1 consisted of patients with PCs and confirmed diagnosis of CBP, whereas group 2 included CBP patients without ultrasound evidence of PCs. Demographic data and clinical history of each assessed patient were reviewed.

\section{Inclusion criteria}

The Inclusion criterion for this study was a diagnosis of 
category II CBP according to National Institutes of Health (NIH) (2) definition and a microbiological assessment of causative pathogens.

\section{Exclusion criteria}

Patients suffering from conditions that influence bacterial virulence or host response (eg. immunodeficiency, abnormalities of the urogenital system) and patients who received antibiotics or immunosuppressive treatment within 4 weeks of the recorded visits were excluded from the study. Patients diagnosed upon investigation with prostatic diseases other than CBP (category I acute bacterial prostatitis, category III chronic prostatitis/chronic pelvic pain syndrome, overt symptomatic benign prostatic hyperplasia, neoplasia) as well as patients exhibiting confounding factors (e.g., indwelling catheters, cystostomy, ureterostomy, ureteral stents, previous prostatic surgery or radiotherapy, incomplete compliance to antibacterial therapy assessed by interviewing patients at the end of treatment) were also excluded.

\section{Patient assessment}

Participants underwent a brief interview in which a complete clinical history was collected. Symptom severity was measured using the National Institutes of Health Chronic Prostatitis Symptom Index (NIH-CPSI) and the International Prostatic Symptoms Score (IPSS) (3).

Urological visits also included digitorectal examination and urine and/or prostatic secretion sample collection, abdominal ultrasound and post-void residual measurement.

\section{Calcification evaluation}

A transrectal ultrasound scan was additionally performed to those who were found with PCs in order to provide both axial and sagittal images, thus improving the evaluation of the number, location, and length of calcifications. TRUS was performed using an $8.0-\mathrm{MHz}$ rectal probe (GE Healthcare, LOGIQ 3). The prostate volume (PV) was measured by TRUS using the formula for an elliptic volume. Besides larger, more echogenic foci that caused acoustic shadowing, also linear calcifications mainly located between transitional and peripheral zone of the gland- were assessed and recorded. Calculi were measured instantly at the time when they were detected on TRUS. A single urologist performed all TRUS procedures and measured calculi.

\section{Microbiological evaluation}

Eligible patients underwent the Meares-Stamey "4-glass" test, based on cultures of first-void (VB1), pre-prostatic massage/midstream (VB2) and post-prostatic massage urine (VB3) specimens, and expressed prostatic secretions (EPS) obtained during prostatic massage (4).

Appropriate antimicrobial agents -accordingly to susceptibility tests- were administered to confirmed cases of CBP for a period of 4 weeks.

Microbiological tests were considered positive when: 1) bacteria grew in the culture of EPS and VB3 specimens and did not in VB1 and VB2; 2) bacterial colonies in VB3 were higher in number compared to VB1 and VB2 specimens. Given that no standard cutoff levels of the num- ber of bacteria in both urine and prostate secretion samples are defined by consensus for the diagnosis of chronic bacterial prostatitis, we defined no lower acceptable level for either one. Cultures, identification and semiquantitative assay for Mycoplasma hominis and Ureaplasma urealyticum were performed using the Mycoplasma IST 2 kit (bioMerieux). Chlamydia trachomatis was detected by direct immunofluorescence, using monoclonal antibodies against lipopolysaccharide membranes (Kallestad). Urine samples were cultured undiluted in blood and MacConkey agar plates (Kallestad Lab., TX, USA) and subjected to centrifugation for microscopic examination of the sediment. Evaluation of culture results was performed by two specialist microbiologists, who were blinded to patient records. Identification of traditional pathogens was performed by conventional methods and the Vitek-2 Compact system (bioMerieux, France), and susceptibility testing was performed by disc diffusion and/or the Vitek-2 system. Interpretation of susceptibility results was based on Clinical and Laboratory Standards Institute (CLSI) guidelines.

\section{Therapy outcome evaluation}

After four weeks of therapy, the NIH-CPSI and IPSS tests were repeated. Follow-up included also interview, physical examination, transrectal ultrasound and the "4-glass" test. The microbiological response to antibacterial therapy was defined in a manner similar to that of Naber et al.: i) eradication: baseline pathogen was eradicated;

ii) persistence: baseline pathogen was not eradicated;

iii) superinfection: baseline pathogen was eradicated with the appearance of a new pathogen (5).

\section{Statistical analysis}

Medians and interquartile ranges (IQR) were used to measure the central tendency and data dispersion of questionnaire ordinal scores. For continuous variables, means and standard deviations were calculated.

The Wilcoxon signed rank test was applied to analyze pre- vs. post-therapy paired differences in NIH-CPSI and IPSS scores, whereas the Wilcoxon rank sum test was used to calculate the significance of differences between different treatment arms at a given time-point. For continuous variables, paired or unpaired t-tests were used to analyze differences between means. All tests were twotailed if not otherwise indicated, and an alpha error inferior to 5\% was set as significance level for each comparison. Comparison between proportions of eradicated patients was made using the Z-test with the Yates' continuity correction.

All inferential calculations were performed using the $\mathrm{R}$ open-source software environment.

\section{RESULTS}

Ninety-five out of 172 eligible patients were assessable at the end of the trial. All patients reported chronic pelvic discomfort and genital pain, with or without lower urinary tract symptoms and sexual dysfunction.

The remaining patients, who were not compliant to therapy or who were lost during follow-up were excluded from the study. 
According to the presence/absence of PCs in ultrasound examination, patients were divided into two groups: 41 individuals had PCs (group 1) and 54 showed no signs of PCs (group 2). The most common symptom in both groups was scrotal/testicular pain (reported by 12 and 17 patients of Group 1 and Group 2 respectively, P > 0.05, Ztest). The most common pathogen in both groups was $E$. Coli (found in 9 and 11 patients of Group 1 and Group 2 respectively $\mathrm{P}>0.05$, Z-test). Data regarding patient demographics, history, clinical symptom presentation and microbiological profiles are listed in Tables 1-3.

No significant differences were found between groups regarding age, marital status, prostate volume and most sexual behaviors. However, a significantly higher number of men with PCs practiced anal penetration (Table 1). Incidentally, the vast majority of these subjects

\section{Table 1.}

Patient demographic and baseline data.

\begin{tabular}{|lccc|}
\hline Clinical sample & Study group & Controls & $\mathbf{p}$ \\
\hline Number of patients & 41 & 54 & - \\
\hline Mean age (years) & 46.8 & 45.1 & $p>0.05$ \\
\hline Chronic prostatitis history (n.) & 37 & 21 & $\mathbf{p}>\mathbf{0 . 0 0 0 1}$ \\
\hline Marital status & & & \\
Married (n.) & 19 & 26 & $p>0.05$ \\
Unmarried (n.) & 7 & 9 & $p>0.05$ \\
Divorced (n.) & 4 & 8 & $p>0.05$ \\
Widower (n.) & 2 & 1 & $p>0.05$ \\
Unknown (n.) & 9 & 10 & \\
\hline Sexual Behaviour & & & \\
Vaginal penetration (n.) & 21 & 24 & $p>0.05$ \\
Anal penetration (n.) & 19 & 16 & $\mathbf{p}=\mathbf{0 . 0 4 7}$ \\
Absence of sexual activity (n.) & 2 & 3 & $p>0.05$ \\
Unknown (n.) & 6 & 11 & $p>0.05$ \\
\hline Mean prostate volume & 40.1 & 39.4 & $p>0.05$ \\
\hline ane-tailed test. & & & \\
\hline
\end{tabular}

Table 3.

Pathogens found in monomicrobial and polymicrobial isolates.

\begin{tabular}{|lccc|}
\hline Isolate & Group 1 & Group 2 & $\begin{array}{c}\text { Intergroup } \\
\text { significance }\end{array}$ \\
\hline Escherichia coli & 13 & 19 & $p>0.05$ \\
\hline Proteus mirabilis & 0 & 5 & na \\
\hline Klebsiella pneumoniae & 2 & 0 & na \\
\hline Morganella morganii & 1 & 0 & na \\
\hline Haemophilus spp. & 1 & 0 & na \\
\hline Acinetobacter baumannii & 1 & 1 & na \\
\hline Enterococcus spp. & 8 & 16 & $p>0.05$ \\
\hline Staphylococcus coagulase-negative & 21 & 21 & $p>0.05$ \\
\hline Streptococcus spp. & 2 & 2 & na \\
\hline na $=$ not assessable. & & & \\
\hline
\end{tabular}

showed enterococcal infections (data not shown). A significantly higher number of men with PCs had a history of chronic prostatitis relapsing episodes (Table 1).

A variety of pathogens were isolated from CBP patients (Table 3). In general, patients showing PCs showed a more

Table 4.

Microbiological and clinical outcomes at the end of therapy.

\begin{tabular}{|lccc|}
\hline Outcome & Group 1 & Group 2 & $\mathbf{p}$ \\
\hline Pathogen eradicated (\%) & $26 / 41(63 \%)$ & $41 / 54(75 \%)$ & $p>0.05$ \\
\hline Pathogen not eradicated & $10 / 41$ & $9 / 54$ & $p>0.05$ \\
\hline Superinfection & $5 / 41$ & $4 / 54$ & $p>0.05$ \\
\hline Clinical resolution & $23 / 41(56.9 \%)$ & $37 / 54(68.5 \%)$ & $p>0.05$ \\
\hline No clinical resolution & $18 / 41$ & $11 / 54$ & $\mathbf{p = 0 . 0 1 2}$ \\
\hline Uncertain clinical resolution & - & $6 / 54$ & - \\
\hline
\end{tabular}

Table 2.

Main and coexisting signs and symptoms in both groups.

\begin{tabular}{|c|c|c|c|}
\hline Group 1 & Group 2 & Main symptom & Frequently assessed coexisting symptoms \\
\hline 12 & 17 & Scrotal/testicular pain & $\begin{array}{l}\text { Perineal and suprapubic pain, haematuria, } \\
\text { frequent urination, nocturia, painful } \\
\text { ejaculation, dysuria, penile pain, erectile } \\
\text { dysfunction }\end{array}$ \\
\hline 5 & 9 & Perineal pain & $\begin{array}{l}\text { Scrotal pain, dysuria, sexual dysfunction, } \\
\text { frequent urination }\end{array}$ \\
\hline 4 & 7 & Dysuria & Scrotal pain, perineal pain, sexual dysfunction \\
\hline 5 & 6 & $\begin{array}{l}\text { Feeling of unusual } \\
\text { heaviness in the scrotum }\end{array}$ & Sexual dysfunction, perineal pain \\
\hline 5 & 4 & Frequent urination & $\begin{array}{l}\text { Testicular pain, mild erectile dysfunction, } \\
\text { burning, suprapubic pain, scrotal pain }\end{array}$ \\
\hline 1 & & Haematospermia & \\
\hline 2 & 4 & Difficult urination & $\begin{array}{l}\text { Frequent urination, scrotal pain, erectile } \\
\text { dysfunction }\end{array}$ \\
\hline 2 & 5 & Penile pain & $\begin{array}{l}\text { Dysuria, scrotal pain, dysuria, feeling of burning, } \\
\text { frequent urination }\end{array}$ \\
\hline 3 & 1 & Suprapubic pain & Dysuria \\
\hline 1 & & $\begin{array}{l}\text { Feeling of burning across } \\
\text { urethra }\end{array}$ & $\begin{array}{l}\text { Frequent urination, scrotal pain, sexual } \\
\text { dysfunction }\end{array}$ \\
\hline 1 & 1 & Local discomfort & \\
\hline
\end{tabular}

diverse variety of Gram-negative pathogens. However, the intergroup proportions of the most frequently isolated species (E. coli, Enterococcus spp., Staphylococcus spp.) were not significantly different. In general, patients showing PC showed a more diverse variety of Gram-negative pathogens.

Microbiological eradication occurred in similar proportions between the two groups (Table 4). Similarly, the resolution of clinical symptoms occurred in equivalent numbers of patients belonging to groups 1 and 2. However, more patients showing no resolution of clinical symptoms belonged to group 1 ( $n=18$ ), compared to group 2 ( $\mathrm{n}=11 ; \mathrm{p}=0.012 ;$ Z-test).

This difference is likely due to the presence of patients showing uncertain resolution of clinical symptoms in group $2(\mathrm{n}=6)$ (Table 4). The latter are absent in group 1. The NIH-CPSI and the IPSS test were used to assess the degree of severity of CBP symptoms in the present study. Paired analysis 
Table 5.

Scores of NIH-CPSI and IPSS symptom questionnaires.

\begin{tabular}{|c|c|c|c|}
\hline Symptom test questionnaire & $\begin{array}{c}\text { Group } 1 \\
\text { score }\end{array}$ & $\begin{array}{c}\text { Group } 2 \\
\text { score }\end{array}$ & p \\
\hline Pre-therapy median NHI-CPSI value (IQR) & 2) $22(9)$ & $19(6)$ & $p=0.036^{b}$ \\
\hline \multirow[t]{2}{*}{ Post-therapy NHI-CPSI value (IQR) } & $10(16)$ & $3(3)$ & $p=0.024^{b}$ \\
\hline & $p<0.0001^{a}$ & $p<0.0001^{a}$ & \\
\hline Pre-therapy median IPSS value (IQR) & $4(9)$ & $4(9)$ & $p=0.91^{b}$ \\
\hline \multirow[t]{2}{*}{ Post-therapy median IPSS value (IQR) } & $1(7)$ & $2(5)$ & $p=0.84^{b}$ \\
\hline & $p<0.0001^{a}$ & $p<0.0001^{a}$ & \\
\hline
\end{tabular}

showed in both groups highly significant improvements of symptoms, assessed with both tests (Table 5). Intergroup analysis resulted in significantly higher scores of the NIHCPSI test in group 1 , both at the pre-therapy and at the post-therapy time points.

Concerning the IPSS test, no differences between groups 1 and 2 were found at both pre- and post-therapy time points (Table 5).

\section{Discussion}

While PCs are a common ultrasound finding, their exact prevalence is not known. It has been reported to vary widely, from $7 \%$ to $70 \%$ with greater incidences occurring in symptomatic conditions $(6,7)$. Differences are mainly due to varieties in the methodology of the studies, as PCs frequency increase with age and in certain conditions, such as prostate hyperplasia and chronic prostatitis. In addition, several studies have strict criteria for prostatic stone definition; thus a large number of cases of prostatic calculi is often overlooked. As mentioned in the methods section, there are two types of echo patterns of calcifications: type I, discrete, multiple small echoes, usually located in the transition and peripheral zones of the prostate or diffusely distributed throughout the gland, and type II, defining large masses of multiple, coarser echoes (8). Some authors consider true stones only those with diameter greater than 3 millimeters $(6,7)$.

In our study the prevalence of PCs among patients with CBP symptoms was about $43 \%$. This finding is similar to that of Shoskes et al., who reported a $46.8 \%$ incidence in a population of relatively younger patients with pelvic pain syndrome (9). However, contrary to our study, these authors excluded cases with stones smaller than 3 $\mathrm{mm}$. On the contrary, Harada et al. defined no limitations on PCs size and found an incidence of $68.8 \%$ in a population of patients with benign prostatic hypertrophy with a higher average age (8). According to autopsy studies, the frequency of PCs in the general population is high and has an increasing age distribution rising up to $99 \%$ in men over 99 years of age (10). Notably, the histological analysis of autopsy material observed histological characteristics of prostatitis in up to $50 \%$ of prostates with calcifications -independently to their size- additionally questioning on the relation between CBP and PCs (10). In fact, the pathophysiology, the clinical relevance and the association of prostatic calculi with prostatic diseases remain unclear.

The exact process of PCs formation is not known. However, it likely involves many factors and according to the origin of the calcification material, PCs may be distinguished in exogenous and endogenous.

The basis for the creation of exogenous PCs is urine reflux to the prostatic ducts as a result of urine flow obstruction. In such case, urine components cause local ionic changes and $\mathrm{pH}$ elevation, which causes the precipitation of salts and the formation of stones. These calculi are usually larger, situated mainly in the prostatic ducts and their composition is similar to stones found anywhere in the urinary tract (11). The basis for the generation of endogenous PCs is the calcification of amyloid particles (a mixture of protein compound rich in lecithin accumulates and degenerated epithelial cells) within the prostatic ducts. This acts as a foreign body, triggering the deposition of calcium and phosphorus salts by epithelial cells (12). A morphological study showed that most PCs (83\%) had bacterial imprints suggesting bacterial colonization and biofilm formation, while another study found DNA and proteins from Escherichia coli in PC bodies $(13,14)$. It is well established that other bacteria such as Gram-positive Enterococcus faecalis and Staphylococcus spp., are also biofilm formers. However, in this study Escherichia coli, Enterococcus faecalis and Staphylococcus spp., were found in equivalent proportions in groups 1 and 2 (Table 3). Of note, Cai et al., performed ultrastructural analysis of prostate biopsy cores obtained from radical prostatectomy specimens and they found prostate calcifications in $60 \%$, positive cultures in $30 \%$ and a structured microbial biofilm in $10 \%$ of the sample (15). As long as biopsy performed for epidemiological purposes provides an instant image of a certain situation, the findings of Cai et al., along with our observations suggests that involvement of pathogenic bacteria follows the formation of calcifications.

As PCs cause mechanical and chemical corrosive effects on the surrounding tissue, the consequent development of fibrosis and edema results in local narrowing of the prostatic ducts, causing stasis of prostatic fluid and new stone formation in a chronic infection process. In such a condition, larger PCs cause greater obstruction, and in this respect Park et al. reported that prostatic inflammatory changes were closely associated with type II calcifications (16).

Given that in most cases PCs are detected incidentally during a random ultrasound check, it is believed that the stones themselves do not usually cause symptoms. However, some researchers demonstrated that the presence of calcifications is more frequently observed in patients with chronic bacterial prostatitis and is related to urinary symptoms (17). Other researchers found significant correlations between the percentage of PCs and the severity of the NIH-CPSI urological symptom subdomain (18). A recent study showed that the presence of PCs may be associated with the severity and worsening of storage symptoms (19), while another recent study showed that PCs plays an important role in sexual dysfunction in middle-aged men with chronic pelvic pain syndrome or chronic prostatitis (20). In the present 
study, significantly higher total scores of the NIH-CPSI test were assessed in group 1, not only before antibacterial treatment, but also after having achieved pathogen eradication (Table 5). This suggests that PC are associated with more severe symptoms of chronic prostatitis, at least in the case of chronically occurring infections. Such increased severity of symptoms is in agreement with the fact that a significantly higher number of patients belonging to group 1 did not show resolution of clinical symptoms at the end of therapy, compared with men without documented calcifications. In contrast to NIHCPSI results, no difference was found between either pre- or post-therapy scores of the IPSS test (Table 5).

In this respect, one should consider that the IPSS test has been tailored for patients with benign prostatic hyperplasia and deals mainly with obstructive voiding symptoms, whereas the NIH-CPSI test also includes pain symptoms, pain scales, irritative symptoms, and a specific quality of life domain. Thus, this latter test is optimal for assessing prostatitis patients, whereas the former is not, though it is often used to complement the NIHCPSI test.

Our results also suggest that the presence of PCs is associated with a previous history of CBP. In fact, PC may serve as a pathogen niche, acting as a source of recurring infection, also caused by biofilm-embedded pathogens, since after treatment the obstructive stones still remain and the inflammatory process continues. Recurrent infection, causing increasing deposition of calcifications, may also occur in patients showing sexual behaviors at high-risk for prostatic infections, like anal sexual intercourse (Table 1).

\section{References}

1. Park B, Choo SH. The burden of prostatic calculi is more important than the presence. Asian J Androl. 2017; 19:482-485.

2. Krieger JN, Nyberg LJr, Nickel JC. NIH consensus definition and classification of prostatitis. JAMA. 1999; 282:236-7.

3. Asvestis C, Varvadesis T, Maravelakis PE. Greek Version of the National Institutes of Health Chronic Prostatitis Symptom Index (NIH-CPSI), its linguistic adaptation and the pilot test of its validity. Hellenic Urol. 2014; 26:1.

4. Stamey TA. Prostatitis. J R Soc Med. 1981; 74:22-40.

5. Naber KG. European Lomefloxacin Prostatitis Study Group. Lomefloxacin versus ciprofloxacin in the treatment of chronic bacterial prostatitis. Int J Antimicrob Agents. 2002; 20:18-27.

6. Geramoutsos I, Gyftopoulos K, Perimenis P, et al. Clinical correlation of prostatic lithiasis with chronic pelvic pain syndromes in young adults. Eur Urol. 2004; 45:333-7.

7. Kim WB, Doo SW, Yang WJ, Song YS. Influence of prostatic calculi on lower urinary tract symptoms in middle-aged men. Urology. 2011; 78:447-9.

8. Harada K, Igari D, Tanahashi Y. Gray scale transrectal ultransonography of the prostate. J Clin Ultrasound. 1979; 7:45-9.

9. Shoskes DA, Lee CT, Murphy D, et al. Incidence and significance of prostatic stones in men with chronic prostatitis/chronic pelvic pain syndrome. Urology. 2007; 70:235-8.

10. Hassler O. Calcifications in the prostate gland and adjacent tis- sues. A combined biophysical and histological study. Pathol. Microbiol. 1968; 31:97-107.

11. Meares EM. Infection stones of the prostate gland. Urology. 1974:4:560-566.

12. Magura CE, Spector M. Scanning electron microscopy of human prostatic corpora amylacea and corpora calculi. Scan Electron Microsc. 1979; 3:713-20.

13. Dessombz A, Méria P, Bazin D, Daudon M. Prostatic stones: evidence of a specific chemistry related to infection and presence of bacterial imprints. PLoS One 2012; 7:e51691.

14. Sfanos KS, Wilson BA, De Marzo AM, Isaacs WB. Acute inflammatory proteins constitute the organic matrix of prostatic corpora amylacea and calculi in men with prostate cancer. Proc Natl Acad Sci USA. 2009; 106:3443-8.

15. Cai T, Tessarolo F, Caola I, et al. Prostate calcifications: A case series supporting the microbial biofilm theory. Investig Clin Urol. 2018; 59:187-193.

16. Park SW, Nam JK, Lee SD, Chung MK. Are prostatic calculi independent predictive factors of lower urinary tract symptoms? Asian J Androl. 2010; 12:221-6.

17. Boltri M, Magri V, Montanari E, et al. Computer-assisted quantitative assessment of prostatic calcifications in patients with chronic prostatitis. Urol Int. 2018; 100:450-455.

18. Engelhardt PF, Seklehner S, Brustmann H, et al. Association between asymptomatic inflammatory prostatitis NIH category IV and prostatic calcification in patients with obstructive benign prostatic hyperplasia. Minerva Urol Nefrol. 2016; 68:242-9.

19. Hyun JS. Clinical Significance of Prostatic Calculi: A Review. World J Mens Health. 2018; 3615-21.

20. Cao JJ, Huang W, Wu HS, et al. Prostatic Calculi: Do They Matter? Sex Med Rev. 2018; 6:482-491.

\section{Correspondence}

Konstantinos Stamatiou, MD (Corresponding Author)

stamatiouk@gmail.com

Richard Lacroix, MD

rlacroix@ureach.com

Nektaria Rekleiti, MD

nekrek@gmail.com

Hippocrates Moschouris, MD

hipmosch@gmail.com

Tzaneio Hospital, Piraeus (Greece)

Vittorio Magri, MD

vittorio.magri@yahoo.it

ASST Nord Milano (Italy)

Gianpaolo Perletti, MD gianpaolo.Perletti@uninsubria.it

Department of Biotechnology and Life Sciences, Section of Medical and Surgical Sciences, University of Insubria, Varese, (taly)

Alberto Trinchieri, $M D$

alberto.trinchieri@gmail.com

CDC Ambrosiana Cesano B, Milano (Italy) 